\title{
Prognostic value of magnetic resonance imaging in the evaluation of physical therapy outcomes in patients with adhesive capsulitis
}

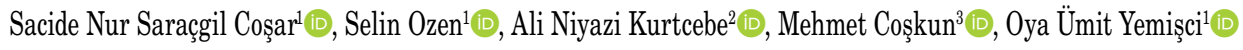 \\ ${ }^{1}$ Department of Physical Medicine and Rehabilitation, Başkent University Faculty of Medicine, Ankara, Turkey \\ ${ }^{2}$ Department of Physical Medicine and Rehabilitation, Gölbaşı Şehit Ahmet Özsoy State Hospital, Ankara, Turkey \\ ${ }^{3}$ Department of Radiology, Başkent University Faculty of Medicine, Ankara, Turkey
}

Received: August 06, 2019 Accepted: April 22, 2020 Published online: May 25, 2021

\begin{abstract}
Objectives: This study aims to investigate whether the treatment outcomes of patients with a clinical diagnosis of adhesive capsulitis (AC) and magnetic resonance imaging (MRI) findings consistent with AC undergoing physical therapy (PT) differs to those with AC in the absence of these MRI findings.

Patients and methods: Between January 2012 and October 2012, a total of 30 patients ( 8 males, 22 females; mean age $55.6 \pm 12.1$ years; range, 35 to 85 years) with a clinical diagnosis of AC underwent MRI of the index shoulder. The MRI scans were evaluated for findings associated with AC: intensity of the inferior glenohumeral ligament (IGHL) and rotator interval (RI). All patients received a total of 15 sessions of PT: hot pack, transcutaneous electrical nerve stimulation, ultrasound, and shoulder exercises. The patients were assessed for shoulder range of motion (ROM) using the Visual Analog Scale (VAS) for shoulder pain, Quick Disabilities of the Arm Shoulder and Hand (Q-DASH), and Health Assessment Questionnaire (HAQ) prior to and following completion of PT.

Results: Of the patients, 21 had no MRI findings consistent with AC (Group 1), nine patients had changes in the IGHL and/or RI characteristic of AC (Group 2). In both groups, there was a significant improvement in active and passive shoulder ROM and VAS scores for shoulder pain at rest (Group 1: $\mathrm{p}<0.001$, Group 2: $\mathrm{p}=0.017$ ) with movement (Group 1: $\mathrm{p}<0.001$, Group 2: $\mathrm{p}=0.007$ ) and at night (Group 1: $\mathrm{p}<0.001$, Group 2: $\mathrm{p}=0.012$ ) following PT. However, there was no significant inter-group difference in scores. Similar findings were recorded for Q-DASH and HAQ.

Conclusion: Based on these study findings, the presence of characteristic MRI findings of AC with a clinical diagnosis do not predict clinical response to physical therapy.
\end{abstract}

Keywords: Adhesive capsulitis, frozen shoulder, physical therapy modality.

Adhesive capsulitis (AC) of the shoulder is a progressive pain syndrome accompanied by decreased active and passive range of motion (ROM) of the glenohumeral joint. ${ }^{[1,2]}$ Adhesive capsulitis has an incidence of 2 to $5 \%$, affecting women more than men and is more commonly seen in the 50 to 70 age group. ${ }^{[3,4]}$ Adhesive capsulitis may be idiopathic or may occur secondary to surgery or trauma and is associated with diabetes, thyroid disease, cerebrovascular disease, autoimmune disease, and Dupuytren's contracture. ${ }^{[5,6]}$ Although the exact pathophysiological process resulting in AC still remains unknown, several histochemical studies have revealed an underlying synovial inflammation, subsequently followed by capsular hypertrophy and reactive fibrosis. ${ }^{[7]}$

The initial clinical diagnosis of AC is characterized by gradual onset of progressive shoulder pain and

Corresponding author: Selin Ozen, MD. Başkent Üniversitesi Tıp Fakültesi Fiziksel Tip ve Rehabilitasyon Anabilim Dalı, 06490 Bahçelievler, Ankara, Türkiye. 
limited ROM which resolves over time. The four stages of the disease reflect this and are comprised of the pre-freezing (0-3 months with limited ROM and pain with active and passive movement), freezing (further limited ROM and chronic pain with active and passive movement at 3-9 months), frozen (severe stiffness, minimal pain for 9-15 months), and thawing (progressive improvement in ROM with minimal pain) stages. ${ }^{[8-11]}$

As AC is a clinical diagnosis, imaging is not mandatory. However, imaging is important in ruling out differential diagnoses such as rotator cuff impingement, calcific tendinitis, osteoarthrosis, and inflammatory arthritis. ${ }^{[12]}$ Magnetic resonance imaging (MRI) is a non-invasive, practical choice of imaging for $\mathrm{AC}$ often preferred in clinical practice. Although AC remains a clinical diagnosis, according to recent literature, the characteristic signs of $\mathrm{AC}$ on MRI can be summarized as the presence of thickening of the coracohumeral ligament (CHL), axillary pouch and rotator interval (RI) joint capsule with a diminished subcoracoid fat triangle. ${ }^{[13]}$ Despite this, a study on characteristic MRI findings and clinical impairment in patients with idiopathic AC of the shoulder has shown no correlation between these two variables. ${ }^{[14]}$

In the early stages of disease, physical therapy (PT) is the first-line treatment. ${ }^{[15]}$ and can be combined with physical modalities such as ultrasound, transcutaneous electrical nerve stimulation (TENS), short-wave diathermy, low-level laser therapy, and hydrotherapy to ease pain and increase ROM. ${ }^{[16]}$ The study by Leung and Cheing ${ }^{[17]}$ showed that the addition of deep heat to stretching exercises in the treatment of AC produced a greater improvement in pain relief, and a better performance in activities of daily living and shoulder ROM, compared to superficial heating. Another study has shown that PT is of most benefit in at least stage two of $\mathrm{AC}$, as the pain is often too severe to permit stretching, ROM and other rehabilitation exercises in stage one of the clinical progression. ${ }^{[18]}$

Considering recent findings showing that characteristic MRI findings in AC do not correlate with the clinical picture, in the present study, we aimed to investigate whether the clinical outcomes of patients with a clinical diagnosis of $\mathrm{AC}$ and typical MRI findings undergoing PT differed to those with AC in the absence of these MRI findings and to determine whether characteristic MRI findings of AC could predict responses to PT.

\section{PATIENTS AND METHODS}

A total of 30 consecutive patients (8 males, 22 females; mean age 55.6 \pm 12.1 years; range, 35 to 85 years) who were admitted to the Physical Medicine and Rehabilitation (PMR) outpatient clinic of Başkent University Ankara Hospital with complaints of unilateral shoulder pain and fulfilled the inclusion and exclusion criteria between January 2012 and October 2012 were included in the study. Inclusion criteria were as follows: unilateral shoulder pain with reduced shoulder ROM in the painful shoulder; and examination findings consistent with the pre-freezing and freezing stages of AC. Exclusion criteria were as follows: contraindications to PT such as the presence of an infection, tumor, uncontrolled hypertension and diabetes, advanced heart or lung disease; contraindications to MRI such as the presence of a cardiac pacemaker, incompatible metallic prosthesis, or claustrophobia; the presence of shoulder dislocation/subluxation, full thickness rotator cuff tear, labral lesion on shoulder MRI; hemiplegia; pregnancy; and intra-articular or subacromial corticosteroid injections to the ipsilateral shoulder within the past three months. Data including age, sex, occupation, level of education, time since onset of symptoms, and past medical history were recorded. Posture, cervical spine and bilateral shoulders were examined. On shoulder examination, active and passive ROM were measured using a goniometer. Shoulder abduction, flexion, and internal and external rotation (with the shoulder abducted to $90^{\circ}$ and the elbow flexed to $90^{\circ}$ ) were measured in the supine position, while shoulder extension was measured with the patient lying face down. Finally, a neurological examination of the upper extremities was performed. All patients were examined by a single PMR specialist prior to treatment and MRI and at the end of the treatment program. Written informed consent was obtained from each patient. The study protocol was approved by the Başkent University Institutional Review Board and Ethics Committee. The study was conducted in accordance with the principles of the Declaration of Helsinki.

\section{Magnetic Resonance Imaging of the shoulder}

After the initial clinical diagnosis of AC was made, the patient underwent MRI (Siemens Magnetom Avanto 1.5T; Siemens AG, Erlangen, Germany) of the symptomatic shoulder. Imaging included T1-weighted turbo spin echo (TSE) and TSE proton density (Pd) frequency selected fat saturation (FS) sequences in 
the axial plane, TSE Pd FS and T2-weighted TSE FS sequences in the coronal plane and T1-weighted TSE and T2-weighted TSE Pd sequences in the sagittal plane.

The MRI was evaluated for findings associated with AC such as thickening and increased intensity of the inferior glenohumeral ligament (IGHL) and RI. The RI is the tendinous triangular space bound by the superior border of the subscapularis inferiorly, the anterior border of the supraspinatus tendon superiorly and the base of the coracoid process medially and contains the long head of the biceps tendon (LHBT). The roof of the RI is formed by the CHL, superior glenohumeral ligament (SGHL) and the RI capsule. ${ }^{[13]}$ The MRI findings were also evaluated with regards to rotator cuff tears, labral lesions, bicipital tendinitis, and periarticular soft tissue and bone pathology. All MRI scans were evaluated by a single radiologist with a special interest in musculoskeletal radiology.

\section{Interventions}

A total of 15 sessions (5 consecutive sessions per week lasting $1 \mathrm{~h}$ each) of hot pack therapy (heated to 71 to $74^{\circ} \mathrm{C}$ for $20 \mathrm{~min}$ ), TENS (at a frequency of $100 \mathrm{~Hz}$, intensity adjusted to the patient's tolerability for a total of $20 \mathrm{~min}$ ) and ultrasound (1 MHz, $1.5 \mathrm{watt} / \mathrm{cm}^{2}$ for a total of $5 \mathrm{~min}$ ) were applied to the anterior, posterior and lateral aspects of the affected shoulder. This was followed by a 20-min session of passive stretching, and passive and active assistive ROM exercises of the shoulder. The patients were also educated for Codman ${ }^{[19]}$ (pendulum), finger ladder, and active shoulder stretching exercises and were asked to repeat two or three times daily in their own time. The treatment was provided by a single physiotherapist with at least 10 years of experience in treating patients with shoulder pathology. The physiotherapist was blinded to the pre- and post-treatment patient evaluation and shoulder MRI findings.

\section{Outcome measures}

The primary outcome measure was shoulder pain at rest, with movement and at night measured using a Visual Analog Scale (VAS). The VAS provides a subjective, visual pain score from $0-100 \mathrm{~mm}$ scored by the patient where $0 \mathrm{~mm}$ is no pain and $100 \mathrm{~mm}$ the worst pain imaginable.

The Quick Disabilities of the Arm Shoulder and Hand (Q-DASH) is an outcome measure of physical function and symptoms related to upper limb musculoskeletal disorders. ${ }^{[20]}$ The Q-DASH is comprised of 11 questions based on activities of daily living (ADLs), social/work activities, levels of pain, and sleep quality. Each item is given a score from one to five and the formula: [(total score/n)-1] $\times 25$ used to calculate a score between 0 and 100 . Higher scores indicate greater symptom severity.

The Health Assessment Questionnaire (HAQ) was initially developed in 1978 by Bruce and Fries $^{[21]}$ to evaluate physical disability in rheumatoid arthritis patients. Later on, it was used to determine physical disability in a number of degenerative joint diseases. ${ }^{[2]}$ The HAQ consists of eight sections each containing 20 items based on ADLs. Each item is given a score between zero and three (0: no difficulty in performing task, 3: cannot perform task). The highest score in each section determines the overall score for that section. The mean score of the eight sections determines the total HAQ score and ranges from zero to three.

All outcome measures were evaluated before the onset of treatment and at the end of the treatment program by a single PMR specialist who was blinded to the shoulder MRI findings.

\section{Statistical analysis}

By taking 30 individuals, with an effect size of 0.5 and Type I error rate as 0.05 and $95 \%$ confidence interval (CI), the achieved power of this study is $0.83 .^{[22]}$ When this study was commenced, there was no prior similar study on which to base a power analysis. Articles on the adequate sample size in initial studies suggest a sample size of between 10 and 30 patients. ${ }^{[23]}$ Therefore, a sample size between 10 to 30 patients per group was required.

Statistical analysis was performed using the SPSS version 11.5 software (SPSS Inc., Chicago, IL, USA). The normal spread of the variables was evaluated analytically using the Shapiro-Wilk test. Categorical variables were expressed in number and percentage, while continuous variables were expressed in mean \pm standard deviation (SD) or median (min-max). For the comparison of the mean and median values between the groups, the Student's t-test and Mann-Whitney U test were used, respectively. Nominal variables were analyzed using the chi-square and Fisher's exact tests. The intra-group differences in pre- and post-treatment outcome measures were evaluated using the Wilcoxon test. The Spearman correlation analysis was performed to evaluate the associations between patient age, time since disease onset, and change in outcome measure values. A two-sided $p$ value of $<0.05$ was considered statistically significant. 


\begin{tabular}{|c|c|c|c|c|c|}
\hline \multicolumn{6}{|c|}{$\begin{array}{c}\text { TABLE } 1 \\
\text { Demographic and clinical characteristics of patients }\end{array}$} \\
\hline & $\mathrm{n}$ & $\%$ & Mean \pm SD & Median & Range \\
\hline Mean age (year) & & & $55.6 \pm 12.1$ & & $35-85$ \\
\hline Sex & & & & & \\
\hline Male & 8 & 26.7 & & & \\
\hline Female & 22 & 73.3 & & & \\
\hline Occupation & & & & & \\
\hline Unemployed & 16 & 53.3 & & & \\
\hline Civil servant & 7 & 23.3 & & & \\
\hline Retired & 3 & 10.0 & & & \\
\hline Other & 4 & 13.3 & & & \\
\hline Symptom duration (month) & & & & 4.5 & $3-48$ \\
\hline Right shoulder & 14 & 46.7 & & & \\
\hline Shoulder trauma & 13 & 43.3 & & & \\
\hline Shoulder injection & 6 & 20 & & & \\
\hline Diabetes mellitus & 6 & 20 & & & \\
\hline Hypertension & 16 & 53.3 & & & \\
\hline Hyperlipidemia & 5 & 16.7 & & & \\
\hline Ischemic heart disease & 4 & 13.3 & & & \\
\hline Thyroid disease & 4 & 13.3 & & & \\
\hline
\end{tabular}

\section{RESULTS}

Baseline demographic and clinical characteristics of patient groups are given in Table 1. The MRI findings revealed thickening and increased intensity of the IGHL in nine $(30 \%)$ patients and an increase in soft tissue signal intensity at the RI in five (16.7\%) patients. A total of nine patients had MRI findings consistent with AC. Pathological MRI findings are summarized in Table 2. A history of shoulder trauma was more common in those without MRI findings consistent with AC (57.1\%), compared to those with

\begin{tabular}{|lcc|}
\hline \multicolumn{4}{|c|}{ TABLE 2 } \\
Pathological findings of shoulder MRI ( $\mathrm{n}=30)$ \\
\hline Findings & $\mathrm{n}$ & $\%$ \\
\hline Change in RI signal intensity & 5 & 16.7 \\
IGHL thickening and increase in signal intensity & 9 & 30 \\
Adhesive capsulitis joint hypertrophy & 19 & 63.3 \\
Degenerative changes of humeral head & 21 & 70.0 \\
Tendinosis & 25 & 83.3 \\
Partial rotator cuff tear & 14 & 46.7 \\
Bursal effusion & 24 & 80.0 \\
\hline $\begin{array}{l}\text { MRI: Magnetic resonance imaging; RI: Rotator interval; } \\
\text { glenohumeral ligament. }\end{array}$ & Inferior \\
\hline
\end{tabular}

MRI findings consistent with AC (11.1\%) $(\mathrm{p}=0.04)$ (Table 3). There was no significant difference in the other demographic and clinical findings of those with MRI findings consistent with AC (change in RI signal intensity and thickening of IGHL), compared to those without (Table 3).

Pre-and post-treatment ROM values for those without MRI findings consistent with AC (Group 1, $\mathrm{n}=21$ ) and those with positive MRI findings consistent with AC (Group 2, n=9) are shown in Table 4. In both groups, there was a significant improvement in post-treatment active and passive ROM in all directions $(\mathrm{p}<0.05)$. However, there was no significant inter-group difference in ROM in any direction after the treatment $(\mathrm{p}>0.05)$.

In both groups, there was a significant improvement in the VAS scores for shoulder pain at rest (Group 1: $\mathrm{p}<0.001$, Group 2: $\mathrm{p}=0.017$ ) with movement (Group 1: $\mathrm{p}<0.001$, Group 2: $\mathrm{p}=0.007$ ) and at night (Group 1: $\mathrm{p}<0.001$, Group 2: $\mathrm{p}=0.012$ ) following PT (Table 5). However, there was no significant inter-group difference in the VAS scores for shoulder pain at rest $(\mathrm{p}=0.1)$, with movement $(\mathrm{p}=0.5)$ and at night $(\mathrm{p}=0.9)$.

In both groups, there was a significant improvement in the HAQ scores (Group 1: $\mathrm{p}<0.001$, 


\section{TABLE 3}

Demographic and clinical findings of those without MRI findings consistent with AC (Group 1) and those with positive MRI findings consistent with AC (Group 2)

\begin{tabular}{|c|c|c|c|c|c|c|c|c|c|c|c|}
\hline & \multicolumn{5}{|c|}{ Group $1(n=21)$} & \multicolumn{5}{|c|}{ Group $2(n=9)$} & \multirow[b]{2}{*}{$\mathrm{n}$} \\
\hline & $\mathrm{n}$ & $\%$ & Mean \pm SD & Median & Range & $\mathrm{n}$ & $\%$ & Mean \pm SD & Median & Range & \\
\hline Age (year) & & & $54.6 \pm 12.7$ & & & & & $58.0 \pm 11.0$ & & & 0.5 \\
\hline Sex & & & & & & & & & & & 0.0 \\
\hline Male & 3 & 14.3 & & & & 5 & 55.6 & & & & \\
\hline Female & 18 & 85.7 & & & & 4 & 44.4 & & & & \\
\hline Time since symptom onset (month) & & & & 6 & $3-48$ & & & & 4 & $3-24$ & 0.4 \\
\hline Right shoulder & 11 & 52.4 & & & & 3 & 33.3 & & & & 0.4 \\
\hline History of shoulder trauma & 12 & 57.1 & & & & 1 & 11.1 & & & & 0.04 \\
\hline
\end{tabular}

\section{TABLE 4}

Pre- and post-treatment shoulder ROM in patient groups

\begin{tabular}{|c|c|c|c|c|c|c|c|c|}
\hline \multirow[b]{2}{*}{ Shoulder movement } & \multicolumn{2}{|c|}{ Pre-treatment $\mathrm{ROM}^{\circ}$} & \multicolumn{2}{|c|}{ Post-treatment $\mathrm{ROM}^{\circ}$} & \multirow[b]{2}{*}{$p^{*}$} & \multicolumn{2}{|c|}{ Change in $\mathrm{ROM}^{\circ}$} & \multirow[b]{2}{*}{$p \dagger$} \\
\hline & Median & Range & Median & Range & & Median & Range & \\
\hline Active abduction & & & & & & & & 0.3 \\
\hline Group 1 & 120 & $50-150$ & 160 & $90-175$ & $<0.001$ & 30 & $5-80$ & \\
\hline Group 2 & 100 & $90-140$ & 150 & $110-170$ & $<0.007$ & 30 & $20-60$ & \\
\hline Active flexion & & & & & & & & 0.4 \\
\hline Group 1 & 120 & $50-150$ & 170 & $100-180$ & $<0.001$ & 30 & $5-90$ & \\
\hline Group 2 & 120 & $80-150$ & 160 & $100-180$ & 0.008 & 40 & $20-60$ & \\
\hline Active extension & & & & & & & & 0.6 \\
\hline Group 1 & 40 & $30-50$ & 55 & $40-60$ & $<0.001$ & 15 & $10-30$ & \\
\hline Group 2 & 30 & $30-40$ & 50 & $40-60$ & 0.007 & 15 & $5-20$ & \\
\hline Active internal rotation & & & & & & & & 0.2 \\
\hline Group 1 & 30 & $15-60$ & 60 & $30-75$ & $<0.001$ & 20 & $5-40$ & \\
\hline Group 2 & 30 & $20-40$ & 50 & $40-70$ & 0.007 & 30 & $10-40$ & \\
\hline Active external rotation & & & & & & & & 0.6 \\
\hline Group 1 & 30 & $15-60$ & 60 & $30-75$ & $<0.001$ & 20 & $5-50$ & \\
\hline Group 2 & 30 & $30-40$ & 50 & $40-70$ & 0.007 & 20 & $10-40$ & \\
\hline Passive abduction & & & & & & & & 0.3 \\
\hline Group 1 & 130 & $70-160$ & 170 & $90-180$ & $<0.001$ & 30 & $10-80$ & \\
\hline Group 2 & 110 & $100-150$ & 170 & $120-180$ & 0.008 & 35 & $15-80$ & \\
\hline Passive flexion & & & & & & & & 0.8 \\
\hline Group 1 & 130 & $90-160$ & 175 & $110-180$ & $<0.001$ & 30 & $10-80$ & \\
\hline Group 2 & 140 & $90-160$ & 180 & $100-180$ & 0.008 & 30 & $10-80$ & \\
\hline Passive extension & & & & & & & & 0.9 \\
\hline Group 1 & 45 & $35-60$ & 60 & $45-60$ & $<0.001$ & 10 & $0-25$ & \\
\hline Group 2 & 45 & $35-50$ & 60 & $45-60$ & 0.007 & 10 & $10-20$ & \\
\hline Passive internal rotation & & & & & & & & 0.5 \\
\hline Group 1 & 40 & $20-70$ & 70 & $35-80$ & $<0.001$ & 20 & $10-45$ & \\
\hline Group 2 & 35 & $30-45$ & 70 & $50-80$ & 0.007 & 30 & $15-40$ & \\
\hline Passive external rotation & & & & & & & & 0.9 \\
\hline Group 1 & 40 & $20-70$ & 70 & $35-80$ & $<0.001$ & 20 & $10-50$ & \\
\hline Group 2 & 40 & $35-45$ & 60 & $45-80$ & 0.007 & 20 & $10-40$ & \\
\hline
\end{tabular}




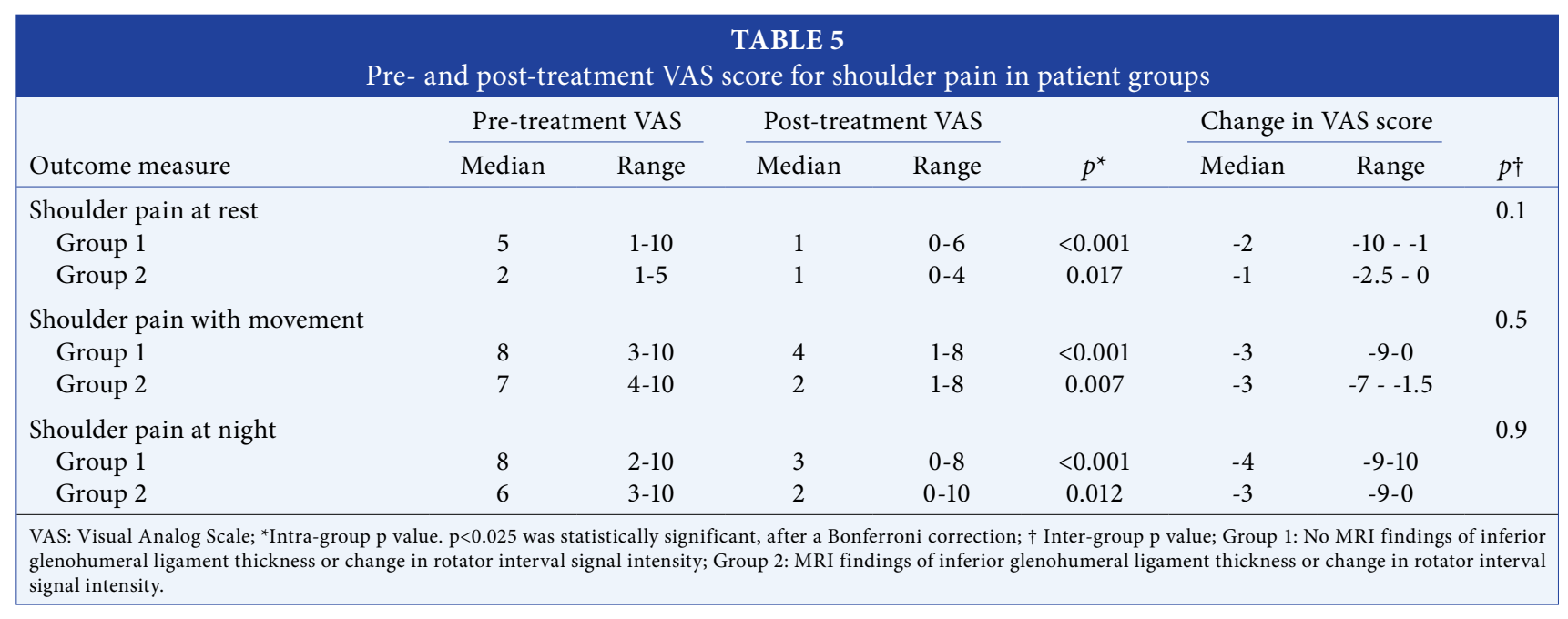

\begin{tabular}{|c|c|c|c|c|c|}
\hline \multicolumn{6}{|c|}{$\begin{array}{c}\text { TABLE } 6 \\
\text { Pre- and post-treatment HAQ and Q-DASH scores in patient groups }\end{array}$} \\
\hline & Pre-treatment score & Post-treatment score & \multirow[b]{2}{*}{$p^{*}$} & \multirow{2}{*}{$\begin{array}{c}\text { Change in score } \\
\text { Mean } \pm \mathrm{SD}\end{array}$} & \multirow[b]{2}{*}{$p^{\dagger}$} \\
\hline & Mean \pm SD & Mean \pm SD & & & \\
\hline HAQ & & & & & 0.4 \\
\hline Group 1 & $1.6 \pm 0.6$ & $1.0 \pm 0.5$ & $<0.001$ & $-0.6 \pm 0.4$ & \\
\hline Group 2 & $1.0 \pm 0.4$ & $0.5 \pm 0.4$ & $<0.001$ & $-0.5 \pm 0.3$ & \\
\hline Q-DASH & & & & & 0.2 \\
\hline Group 1 & $63.1 \pm 18.6$ & $38.6 \pm 18.7$ & $<0.001$ & $-24.5 \pm 15.0$ & \\
\hline Group 2 & $38.6 \pm 17.0$ & $22.2 \pm 21.0$ & 0.003 & $-16.4 \pm 12.0$ & \\
\hline $\begin{array}{l}\text { HAQ: Health } \\
\text { significant, aft } \\
\text { in rotator inter }\end{array}$ & $\begin{array}{l}\text { tionnaire; Q-DASH: Quick } \\
\text { correction; } \dagger \text { Inter-group } ~ \\
\text { ity; Group 2: MRI finding }\end{array}$ & $\begin{array}{l}\text { abilities of the Arm Shoulder } \\
\text { ue; Group 1: No MRI findin } \\
\text { nferior glenohumeral ligame }\end{array}$ & $\begin{array}{l}\mathrm{d} \text { Hand; }{ }^{*} \\
\text { of inferior }\end{array}$ & $\begin{array}{l}\text { group } \mathrm{p} \text { value. } \mathrm{p}<0.025 \\
\text { humeral ligament thick } \\
\text { inge in rotator interval s }\end{array}$ & $\begin{array}{l}\text { isticall } \\
\text { chang } \\
\text { ntensity }\end{array}$ \\
\hline
\end{tabular}

\begin{tabular}{|c|c|c|c|c|}
\hline \multicolumn{5}{|c|}{$\begin{array}{l}\text { TABLE } 7 \\
\text { Correlation between patient age, time since disease onset, and improvement in outcome measures } \\
\text { following physical therapy }\end{array}$} \\
\hline & \multicolumn{2}{|c|}{ Age (year) } & \multicolumn{2}{|c|}{ Time since symptom onset (month) } \\
\hline & $\mathrm{r}$ value & $p^{*}$ & $\mathrm{r}$ value & $p$ \\
\hline Active shoulder abduction & 0.07 & 0.7 & 0.03 & 0.9 \\
\hline Active shoulder flexion & 0.1 & 0.5 & 0.1 & 0.6 \\
\hline Active shoulder extension & -0.2 & 0.2 & -0.02 & 0.9 \\
\hline Active shoulder internal rotation & -0.1 & 0.5 & 0.3 & 0.1 \\
\hline Active shoulder external rotation & -0.09 & 0.6 & 0.4 & 0.03 \\
\hline Passive shoulder abduction & -0.06 & 0.8 & 0.01 & 1.0 \\
\hline Passive shoulder flexion & 0.06 & 0.7 & -0.1 & 0.6 \\
\hline Passive shoulder extension & -0.08 & 0.7 & -0.3 & 0.06 \\
\hline Passive shoulder internal rotation & -0.2 & 0.3 & 0.3 & 0.08 \\
\hline Passive shoulder external rotation & -0.2 & 0.4 & 0.4 & 0.02 \\
\hline VAS for shoulder pain at rest & -0.3 & 0.1 & -0.2 & 0.3 \\
\hline VAS for shoulder pain with movement & -0.3 & 0.07 & -0.3 & 0.1 \\
\hline VAS for shoulder pain at night & -0.2 & 0.2 & -0.4 & 0.02 \\
\hline HAQ & -0.2 & 0.3 & -0.2 & 0.2 \\
\hline Quick DASH & -0.5 & 0.01 & -0.3 & 0.06 \\
\hline
\end{tabular}


Group 2: $<0.001)$ with PT. However, there was no significant inter-group difference in the HAQ scores $(\mathrm{p}=0.4)$ (Table 6). Equally, in both groups, there was a significant improvement in the Q-DASH scores (Group 1: $\mathrm{p}<0.001$, Group 2: $\mathrm{p}=0.003$ ) with PT. However, there was no significant inter-group difference in the Q-DASH scores $(\mathrm{p}=0.2)$ (Table 6).

There was an inverse relationship between age and Q-DASH scores following treatment; as the age of the patient increased, there was a greater reduction in the Q-DASH scores $(\mathrm{r}=-0.5, \mathrm{p}=0.01)$ (Table 7). There was a direct correlation between the duration of symptoms and active and passive external rotation $(\mathrm{p}=0.03$ and $\mathrm{p}=0.02$, respectively). There was an inverse correlation between the duration of symptoms and VAS for shoulder pain at night $(\mathrm{r}=-0.4, \mathrm{p}=0.02)$. Therefore, those with a longer duration of symptoms obtained a greater improvement in active and passive shoulder external rotation with treatment and their shoulder pain at night reduced significantly.

\section{DISCUSSION}

The diagnosis of shoulder AC has traditionally been made based on clinical findings. In more recent years, shoulder MRI has been used as an adjunct to clinical diagnosis and recent studies have suggested that MRI can be used in the clinical staging of AC. ${ }^{[24]}$ In the light of these data, the present study investigated the predictive value of MRI findings consistent with AC for the clinical outcome of PT. In this study, both those with and without MRI findings consistent with AC showed a significant improvement in active and passive ROM, VAS for shoulder pain, HAQ, and Q-DASH. However, inter-group differences were not significant. This suggests that patients with a clinical diagnosis of $\mathrm{AC}$ benefit from $\mathrm{PT}$, regardless of whether or not shoulder MRI findings also support the diagnosis. In addition, these results suggest that MRI findings consistent with a diagnosis of shoulder AC do not predict treatment outcomes with regards to pain, shoulder movement, functionality, and quality of life. When the correlation between patient age, time since disease onset, and improvement in outcome measures following PT was analyzed, disability of the arm shoulder and hand reduced more in the older patients who received PT. The natural clinical progression of AC over time with $\mathrm{PT}$ may have contributed to the findings of a direct correlation between longevity of symptoms and increased ROM and reduction in shoulder pain at night.
In this study, thickening and increased intensity of the IGHL and rotator interval RI were evaluated due to their association with $\mathrm{AC}$ based on the findings of previous studies. In a study, Gondim Teixeira et al. ${ }^{[25]}$ compared shoulder MRIs of 34 patients with a clinical diagnosis of AC with those of an age-matched control group with no signs of AC. The T2 hyperintensity of the IGHL showed a high sensitivity (85.3 to $88.2 \%)$ and specificity (88.2\%) for the diagnosis of AC with an excellent interobserver reliability $(\kappa=0.85)$. Similarly, in the study by Lefevre-Colau et al., ${ }^{[26]}$ in which MRI findings of AC shoulders were compared to pain-free contralateral shoulders, the interobserver reliability of assessment of the RI was deemed acceptable.

The study by Park et al. ${ }^{[14]}$ investigated the correlation between characteristic shoulder MRI findings consistent with AC and clinical impairment in patients with idiopathic AC. The authors found no correlation between thickness of the axillary recess, CHL and enhanced portion of the RCI and shoulder pain, active and passive shoulder ROM, and Constant-Murley score. In contrast, a very recent study by Chellathurai et al. ${ }^{[24]}$ found that particularly thickening of the anterior band of IGHL was significantly correlated with the clinical staging of AC with the distribution of edema of IGHL and pericapsular edema also showing significant correlation. The authors of the study showed the difference in findings to Park et al.'s ${ }^{[14]}$ study down to the fact that they did not evaluate the anterior band of IGHL and concluded that MRI could be useful in both evaluating and predicting the clinical staging of AC.

Previous studies have shown that often other shoulder pathologies such as tendinosis, subdeltoid subacromial bursitis, partial and total rotator cuff tears are found to accompany $\mathrm{AC}$ on evaluation of shoulder MRI. ${ }^{[27-30]}$ In the present study, AC joint hypertrophy, degenerative changes of humeral head, tendinosis, partial rotator cuff tear, and bursal effusions were present in 43.7 to $86.3 \%$ of patients. The presence of such pathologies in addition to findings associated with AC may have been one reason why MRI findings did not seem to predict the outcome of treatment; the presence of other pathologies would also affect the response to treatment. However, this situation is a reflection of the clinical reality; more often than not, patients with clinical findings consistent with AC will also have other shoulder joint pathologies which may also influence PT outcomes. Presence of concomitant pathologies rather than $\mathrm{AC}$ alone maybe one reason why MRI did not seem to predict the outcome of treatment. 
To the best of our knowledge, this is the first, prospective study investigating the prognostic value of MRI in the treatment of AC with PT in the literature. In this study, we attempted to provide a holistic evaluation of treatment outcome by measuring ROM, and questioning shoulder pain, functionality, and quality of life. The study limitations included: (i) the presence of other shoulder pathologies on MRI; (ii) MRI evaluation by a single radiologist; (iii) lack of imaging of the contralateral shoulder as a control; (iv) the relatively small sample size; and (v) the small number of patients with MRI findings consistent with AC. Future studies may include a more detailed analysis of MRI findings including edema of the IGHL in the humeral and glenoid portions of the axillary pouch, anterior and posterior pericapsular edema, obliteration of the fat in the subcoracoid triangle to identify the correlation between such positive findings and treatment outcomes. Furthermore, outcomes of a PT program based on the MRI clinical staging of AC can be compared with the outcomes of a PT program based on clinical findings.

In conclusion, our study results suggest that MRI of the shoulder can be used to rule out the differential diagnoses of AC. However, the presence of pathological findings of IGHL and RI, which support a clinical diagnosis of AC, do not predict outcomes of treatment of AC with PT.

\section{Declaration of conflicting interests}

The authors declared no conflicts of interest with respect to the authorship and/or publication of this article.

\section{Funding}

The authors received no financial support for the research and/or authorship of this article.

\section{REFERENCES}

1. Emig EW, Schweitzer ME, Karasick D, Lubowitz J. Adhesive capsulitis of the shoulder: MR diagnosis. AJR Am J Roentgenol 1995;164:1457-9.

2. Sofka CM, Ciavarra GA, Hannafin JA, Cordasco FA, Potter HG. Magnetic resonance imaging of adhesive capsulitis: correlation with clinical staging. HSS J 2008;4:164-9.

3. Bunker TD. Frozen shoulder: unravelling the enigma. Ann R Coll Surg Engl 1997;79:210-3.

4. Reeves B. The natural history of the frozen shoulder syndrome. Scand J Rheumatol 1975;4:193-6.

5. D'Orsi GM, Via AG, Frizziero A, Oliva F. Treatment of adhesive capsulitis: a review. Muscles Ligaments Tendons J 2012;2:70-8.

6. Harris JD, Griesser MJ, Copelan A, Jones GL. Treatment of adhesive capsulitis with intra-articular hyaluronate: A systematic review. Int J Shoulder Surg 2011;5:31-7.
7. Brue S, Valentin A, Forssblad M, Werner S, Mikkelsen C, Cerulli G. Idiopathic adhesive capsulitis of the shoulder: a review. Knee Surg Sports Traumatol Arthrosc 2007;15:1048-54.

8. Hannafin JA, Chiaia TA. Adhesive capsulitis. A treatment approach. Clin Orthop Relat Res 2000;372:95-109.

9. Neviaser JS. Adhesive capsulitis of the shoulder: a study of the pathological findings in periarthritis of the shoulder. J Bone Joint Surg 1945;27:211-22.

10. Neviaser RJ, Neviaser TJ. The frozen shoulder. Diagnosis and management. Clin Orthop Relat Res 1987;223:59-64.

11. Neviaser RJ. Painful conditions affecting the shoulder. Clin Orthop Relat Res 1983;173:63-9.

12. Gordon JA, Breitbart E, Austin DC, Photopoulos CD, Kelly JD. Adhesive capsulitis: diagnosis, etiology, and treatment strategies. In: Kelly JD, editor. Elite Techniques in Shoulder Arthroscopy: New Frontiers in Shoulder Preservation. Cham: Springer; 2016. p. 149-68.

13. Fields BKK, Skalski MR, Patel DB, White EA, Tomasian A, Gross JS, et al. Adhesive capsulitis: review of imaging findings, pathophysiology, clinical presentation, and treatment options. Skeletal Radiol 2019;48:1171-84.

14. Park GY, Park JH, Kwon DR, Kwon DG, Park J. Do the Findings of Magnetic Resonance Imaging, Arthrography, and Ultrasonography Reflect Clinical Impairment in Patients With Idiopathic Adhesive Capsulitis of the Shoulder? Arch Phys Med Rehabil 2017;98:1995-2001.

15. Le HV, Lee SJ, Nazarian A, Rodriguez EK. Adhesive capsulitis of the shoulder: review of pathophysiology and current clinical treatments. Shoulder Elbow 2017;9:75-84.

16. Hsu JE, Anakwenze OA, Warrender WJ, Abboud JA. Current review of adhesive capsulitis. J Shoulder Elbow Surg 2011;20:502-14.

17. Leung MS, Cheing GL. Effects of deep and superficial heating in the management of frozen shoulder. J Rehabil Med 2008;40:145-50.

18. Sheridan MA, Hannafin JA. Upper extremity: emphasis on frozen shoulder. Orthop Clin North Am 2006;37:531-9.

19. Codman EA. The Shoulder: Rupture of the Supraspinatus Tendon and Other Lesions in or About the Subacromial Bursa. 1st ed. Malabar, FL: G. Miller and Co Medical Publishers Inc; 1984.

20. Beaton DE, Wright JG, Katz JN; Upper Extremity Collaborative Group. Development of the QuickDASH: comparison of three item-reduction approaches. J Bone Joint Surg Am 2005;87:1038-46.

21. Bruce B, Fries JF. The Health Assessment Questionnaire (HAQ). Clin Exp Rheumatol 2005;23(5 Suppl 39):S14-8.

22. Faul F, Erdfelder E, Buchner A, Lang AG. Statistical power analyses using $G^{\star}$ Power 3.1: tests for correlation and regression analyses. Behav Res Methods 2009;41:1149-60.

23. Johanson GA, Brooks GP. Initial Scale Development: Sample Size for Pilot Studies. Educational and Psychological Measurement 2010;70:394-400.

24. Chellathurai A, Subbiah K, Elangovan A, Kannappan S. Adhesive capsulitis: MRI correlation with clinical stages and proposal of MRI staging. Indian J Radiol Imaging 2019;29:19-24. 
25. Gondim Teixeira PA, Balaj C, Chanson A, Lecocq S, Louis M, Blum A. Adhesive capsulitis of the shoulder: value of inferior glenohumeral ligament signal changes on T2-weighted fat-saturated images. AJR Am J Roentgenol 2012;198:W589-96.

26. Lefevre-Colau MM, Drapé JL, Fayad F, Rannou F, Diche T, Minvielle F, et al. Magnetic resonance imaging of shoulders with idiopathic adhesive capsulitis: reliability of measures. Eur Radiol 2005;15:2415-22.

27. Connell D, Padmanabhan R, Buchbinder R. Adhesive capsulitis: role of $\mathrm{MR}$ imaging in differential diagnosis. Eur Radiol 2002;12:2100-6.
28. Tamai K, Yamato M. Abnormal synovium in the frozen shoulder: a preliminary report with dynamic magnetic resonance imaging. J Shoulder Elbow Surg 1997;6:53443.

29. Carrillon Y, Noel E, Fantino O, Perrin-Fayolle O, TranMinh VA. Magnetic resonance imaging findings in idiopathic adhesive capsulitis of the shoulder. Rev Rhum Engl Ed 1999;66:201-6.

30. Yoo JC, Ahn JH, Lee YS, Koh KH. Magnetic resonance arthrographic findings of presumed stage-2 adhesive capsulitis: focus on combined rotator cuff pathology. Orthopedics 2009;32:22. 